\title{
A Comparison of Wavelength Dependence for Laser-assisted Lipolysis Effect Using Monte Carlo Simulation
}

\author{
Jong-In Youn* \\ Department of Biomedical Engineering, Catholic University of Daegu, Gyeongsan 712-702, Korea
}

(Received April 1, 2009 : revised June 8, 2009 : accepted June 8, 2009)

\begin{abstract}
The aim of this study is to evaluate wavelength dependence for laser-assisted lipolysis using a mathematical simulation. In this study, a Monte Carlo simulation was performed to simulate light transport in fat and dermal tissue with 3 different laser wavelengths $(\lambda=1064 \mathrm{~nm}, 1320 \mathrm{~nm}$, and $1444 \mathrm{~nm}$ ) that are currently used in clinic settings for laser-assisted lipolysis. The relative rates of heat generation versus penetration depth showed that the greatest amount of heat generation was seen in the tissues at $\lambda=1444 \mathrm{~nm}$. This Monte Carlo simulation may help lend insight into the thermal events occurring inside the fat and dermal tissue during laser-assisted lipolysis.
\end{abstract}

Keywords: Dermis, Fat, Laser-assisted lipolysis, Monte Carlo simulation, Wavelength dependence

OCIS codes : (170.0170) Medical optics and biotechnology; (000.1430) Biology and medicine; (030.5290) Photon statistics; (170.1870) Dermatology

\section{INTRODUCTION}

As more women and men want to look and feel better about themselves, body contouring and liposculpture continue to grow in popularity, making laser lipolysis one of the most popular cosmetic surgeries performed today [1-4]. Laser lipolysis is usually performed with a cannula that contains a laser fiber. The cannula is inserted into the treatment area and moved back and forth so that the laser energy can dissolve unwanted fat. The immediate effect of laser-assisted lipolysis is to produce temporary pores in the cell membranes of adipocytes, allowing dissolved fat to migrate into the extracellular space [1-4]. Laser lipolysis causes less bleeding, bruising, and swelling than conventional liposuction procedures, resulting in a faster recovery [1-4].

Currently, laser lipolysis is performed in the clinical setting with a Nd:YAG laser at two different wavelengths, $\lambda=1064 \mathrm{~nm}$ (Smartlipo ${ }^{\mathrm{TM}}$, Cynosure, Inc., Westford, MA; LipoLite ${ }^{\mathrm{TM}}$, Syneron Inc., Irvine CA) and $\lambda=1320$ nm (CoolLipo ${ }^{\mathrm{TM}}$, CoolTouch Corp., Roseville, CA). In this study, a wavelength dependence modeling of the laser lipolysis effect was performed using the two popular laser wavelengths in the market, $\lambda=1064 \mathrm{~nm}$ and 1320 $\mathrm{nm}$, and the recently introduced novel laser wavelength,

*Corresponding author: jyoun@cu.ac.kr $\lambda=1444 \mathrm{~nm}$ (AccuSculpt ${ }^{\mathrm{TM}}$, Lutronic Corp., South Korea). The recent preliminary measurements showed that the amount of removed mass after laser irradiation at $28 \mathrm{~kJ} / \mathrm{cm}^{2}$ for these three different laser wavelengths, $1064 \mathrm{~nm}, 1320 \mathrm{~nm}$, and $1444 \mathrm{~nm}$, were $15.46 \mathrm{mg}, 24.78$ $\mathrm{mg}$, and $45.19 \mathrm{mg}$, respectively [5]. From the results, $\lambda=1444 \mathrm{~nm}$ provided the highest ablation efficiency as compared with the other two laser wavelengths, $\lambda$ $=1064 \mathrm{~nm}$ and $1320 \mathrm{~nm}$. In this study, wavelength dependence study for laser-assisted lipolysis evaluated using a mathematical method, Monte Carlo simulation, was performed to evaluate the heat generation as a function of depth during the laser-assisted lipolysis procedure at the three different laser wavelengths for the better understanding of thermodynamic mechanisms in fat and dermis.

The Monte Carlo simulation technique, which is based on the statistical nature of radiation interactions, has been widely applied in laser radiation transport studies [6-12]. Monte Carlo simulation methods trace the propagation of individual photons through a turbid medium until the photon experiences complete absorption by or escape from the medium [6]. In this study, the heat generation in two different homogeneous regions, fat and dermal tissue, was investigated by a computer simulation of appropriately weighted random absorption and scattering interactions. This mathematical modeling may 


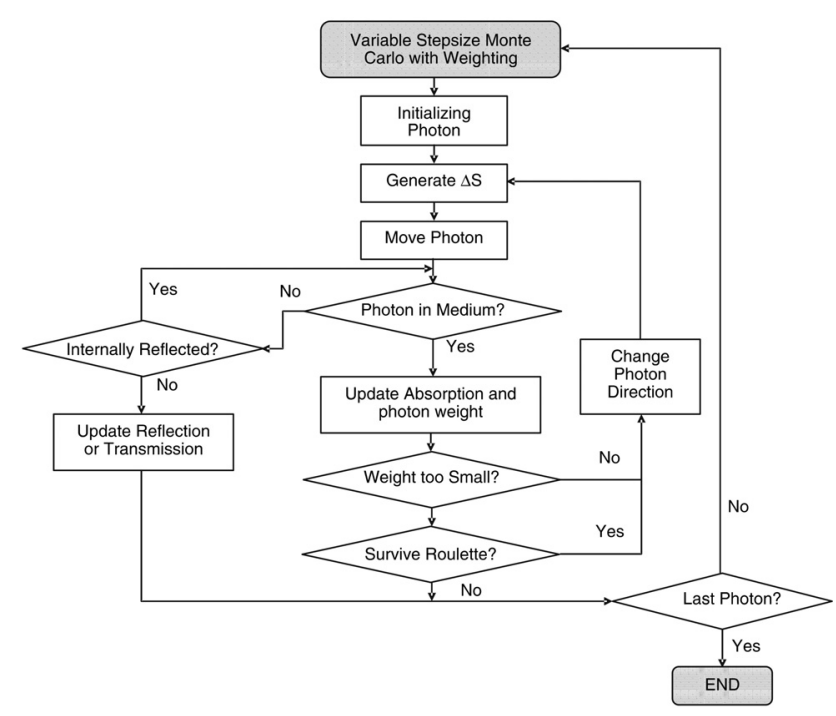

FIG. 1. Variable stepsize Monte Carlo with weighting.

provide a better understanding of the laser lipolysis process and help to determine the most effective wavelength for the fat volume to be removed.

\section{MONTE CARLO SIMULATION METHODS}

In this study, a Monte Carlo model of laser light distribution in tissue was coded in ANSI standard C. The model is based on calculations describing the heat generation using three different wavelengths of laser light $(\lambda$ $=1064 \mathrm{~nm}, 1320 \mathrm{~nm}$, and $1444 \mathrm{~nm}$ ).

Figure 1 shows a flowchart of a Monte Carlo simulation that uses a weighted photon to improve the statistics of the simulation. Each generated photon is moved a distance, $\Delta s$, where it may be scattered, absorbed, propagated undisturbed, internally reflected, or transmitted out of the tissue [7-10].

\section{Initializing the photon}

Each photon is initially assigned a weight, $W$, that is initialized to 1 . When a photon is launched, the tissue surface is first checked as to whether or not there is a refractive-index mismatched boundary. Some photons may be specularly reflected, in which case the photon weight is decreased [6].

The specular reflectance is specified as:

$$
R_{s p}=\frac{\left(n_{1}-n_{2}\right)^{2}}{\left(n_{1}+n_{2}\right)^{2}}
$$

The photon weight is decreased by $R_{s p}$ for the photon to enter the tissue:

$$
W=1-R_{s p}
$$

\section{Photon Moving}

The stepsize of each launched photon is calculated based on a random sampling of the probability distribution for the photon's free path. The computer's random number generator yields a random variable, $\boldsymbol{S}$, from 0 to $1[6]$. The stepsize is determined by:

$$
s=\frac{-\ln \varsigma}{\mu_{t}}
$$

Once stepsize is determined, the photon is moved in the tissue. For the photon located at $(x, y, z)$ traveling a stepsize distance in the direction $\left(\mu_{x}, \mu_{y}, \mu_{z}\right)$, the new coordinates $\left(\mathrm{x}^{\prime}, \mathrm{y}^{\prime}, \mathrm{z}^{\prime}\right)$ are determined by:

$$
\begin{aligned}
& x^{\prime}=x+\mu_{x} s \\
& y^{\prime}=y+\mu_{y} s \\
& z^{\prime}=z+\mu_{z} s
\end{aligned}
$$

\section{Internal reflectance at the boundary}

During each stepsize movement, the photon may hit a boundary. The photon can be either internally reflected by the boundary or escape as observed reflectance. The probability that the photon will be internally reflected is determined by Fresnel's law [6]:

$$
R\left(\theta_{i}\right)=\frac{1}{2}\left\{\frac{\sin ^{2}\left(\theta_{i}-\theta_{t}\right)}{\sin ^{2}\left(\theta_{i}+\theta_{t}\right)}+\frac{\tan ^{2}\left(\theta_{i}-\theta_{t}\right)}{\tan ^{2}\left(\theta_{i}+\theta_{t}\right)}\right\}
$$

Therefore, photon weight that successfully escapes from the tissue is described as:

$$
1-R\left(\theta_{i}\right)
$$

\section{Photon absorption}

Once the photon has reached an interaction site, some attenuation of the photon weight owing to absorption by the tissue may occur. The new photon weight is calculated by:

$$
W \leftarrow W \frac{\mu_{s}}{\mu_{t}}
$$

When photon weight is below the threshold weight, the photon weight becomes 0 and the photon is terminated. However, propagating a photon with a minuscule weight yields little information and the roulette technique gives the photon one chance of surviving with a weight of $\mathrm{mW}(\mathrm{m}=10)$.

\section{Photon scattering}

When an anisotropy factor $(\mathrm{g})$ value is 0 , indicating isotropic scattering, the cosine of the deflection angle is described as: 
Tiny MC(dermis)

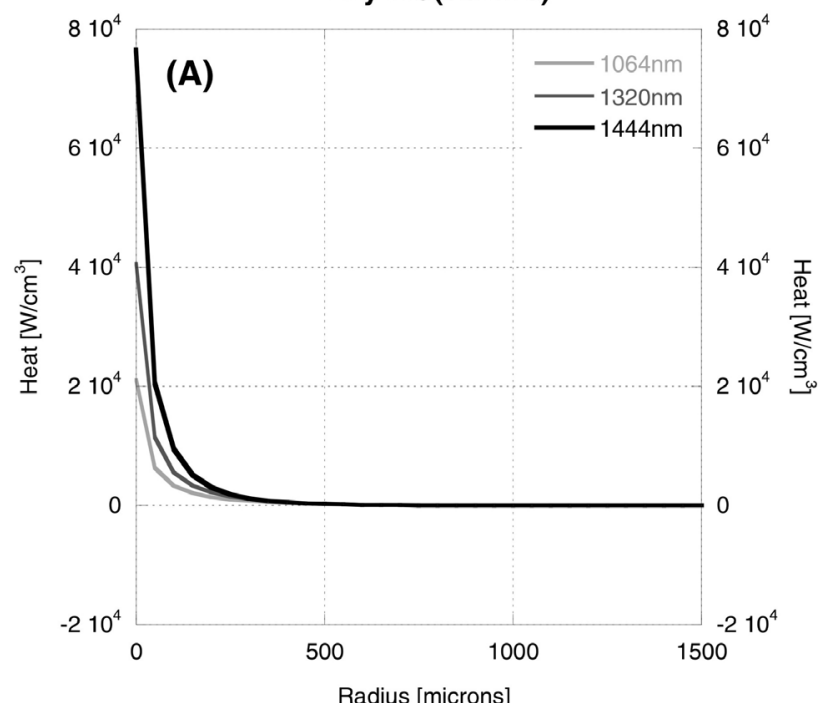

Small MC(dermis)

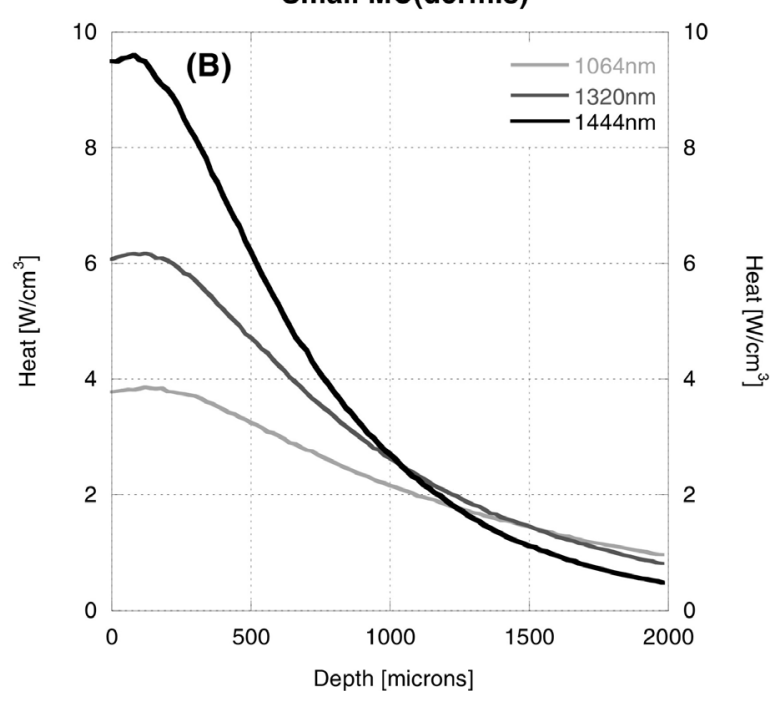

FIG. 3. Relative heat generation vs. penetration depth in the dermis using Monte Carlo (MC) simulations; (upper) Tiny MC, (lower) Small MC.

TABLE 1. Optical properties of fat and dermal tissue for Monte Carlo simulation variables.

\begin{tabular}{c|c|c|c|c}
\hline \hline Wavelength & $\lambda_{(\mathrm{nm})}$ & $1064 \mathrm{~nm}$ & $1320 \mathrm{~nm}$ & $1444 \mathrm{~nm}$ \\
\hline \multirow{4}{*}{ Fat } & $\mu_{a}\left[\mathrm{~cm}^{-1}\right]$ & 1.5 & 2 & 2.5 \\
\cline { 2 - 5 } & $\mu_{S}\left[\mathrm{~cm}^{-1}\right]$ & 11 & 10.5 & 11 \\
\cline { 2 - 5 } & $\mathrm{n}$ & & 1.37 & \\
\cline { 2 - 5 } & $\mathrm{g}$ & & 0.91 & \\
\hline \multirow{5}{*}{ Dermis } & $\mu_{a}\left[\mathrm{~cm}^{-1}\right]$ & 1 & 2 & 4 \\
\cline { 2 - 5 } & $\mu_{S}\left[\mathrm{~cm}^{-1}\right]$ & 250 & 250 & 250 \\
\cline { 2 - 5 } & $\mathrm{n}$ & & 1.36 & \\
\cline { 2 - 5 } & $\mathrm{g}$ & & 0.91 & \\
\hline
\end{tabular}

Photon \# 10000

Abbreviations: $\mathrm{n}$, refractive index, g, anisotropic factor.

$$
\cos \theta=2 \varsigma-1
$$

When an anisotropy factor $(\mathrm{g})$ value is near 1 , indicating anisotropic scattering, the cosine of the deflection angle is described as:

$$
\cos \theta=\frac{1}{2 g}\left\{1+g^{2}-\left(\frac{1-g^{2}}{1-g+2 g \varsigma}\right)^{2}\right\}
$$

This process is repeated according to the total number of photons [3-6].

In this study, two different simulation algorithms were used, as described below, at three laser wavelengths:

\section{- Tiny Monte Carlo Simulation}

Simulates light propagation from a point source in an infinite medium with isotopic scattering and calculates the heating as a function of radius [7, 8].

- Small Monte Carlo Simulation.

Simulates light propagation from normal irradiation of a semi-infinite medium with anisotropic scattering and calculates the volumetric heating as a function of depth $[7,8]$.

Heat generation is calculated by $[6,11]$ :

$$
\text { Heat }\left[W / \mathrm{cm}^{3}\right]=\mu_{a} \frac{3}{4 \pi r} \mu_{t}{ }^{\prime} P_{0} e^{-\sqrt{\mu_{a} \mu_{t}}}
$$

where $\mu_{a}, r, \mu_{t}^{\prime}, P_{0}$ are the absorption coefficient, the radial distance, the total attenuation coefficient and total radiant power, respectively.

Using the heat generation equation (10) and the various steps in Figure 1, the tiny and small Monte Carlo simulations are applied to fat and dermal tissue.

Table 1 shows the optical properties of fat and dermal tissue for Monte Carlo simulation variables [9, 10].

\section{RESULTS AND DISCUSSION}

Tiny Monte Carlo simulation and Small Monte Carlo simulation are performed with fat and dermal tissue.

Figure 2 shows the relative rate of heat generation versus optical penetration depth in fat tissue for the three different wavelengths $(\lambda=1064 \mathrm{~nm}, 1320 \mathrm{~nm}$, and $1444 \mathrm{~nm}$ ). For both Tiny Monte Carlo and Small Monte Carlo simulations, the rate of heat generation at the surface is greater than the rate in the deep fat 
layers. For Tiny Monte Carlo simulation, the heat is dramatically reduced as the penetration depth is increased.

These results demonstrated that the highest heat generation in fat tissue was achieved at $\lambda=1444 \mathrm{~nm}$, followed by, in order, $\lambda=1320 \mathrm{~nm}$ and $1064 \mathrm{~nm}$. However, the heat generation at $\lambda=1444 \mathrm{~nm}$ was less than that at $\lambda=1064 \mathrm{~nm}$ below a depth of $3.5 \mathrm{~mm}$. According to this result, the localized maximum heat generation for fat tissue can be achieved at $\lambda=1444 \mathrm{~nm}$, with minimal damage of the surrounding region.

Figure 3 presents the results from Tiny Monte Carlo and Small Monte Carlo simulations of the dermis. As with Monte Carlo simulations of fat tissue (Figure 2), the highest heat generation was achieved at $\lambda=1444$ $\mathrm{nm}$, followed by, in order, $\lambda=1320 \mathrm{~nm}$ and $1064 \mathrm{~nm}$. In the same manner, localized maximum heat generation for dermal tissue can be achieved at $\lambda=1444 \mathrm{~nm}$, with minimal damage of the surrounding region because the heat generation at $\lambda=1444 \mathrm{~nm}$ is less than that at $\lambda$ $=1064 \mathrm{~nm}$ below a depth of $1.2 \mathrm{~mm}$.

Laser lipolysis is performed by inserting a cannula into the fat layer under the skin. There is little likelihood that the procedure results in thermal or mechanical damage to the dermis. The results from Monte Carlo simulation for both fat and dermal tissue suggest that any injury will be greater with the 1444-nm laser than with the other two wavelengths, $\lambda=1064 \mathrm{~nm}$ and 1320 $\mathrm{nm}$. However, the minimal localization of heat at $\lambda=$ $1444 \mathrm{~nm}$ for both fat and dermal tissue using laserassisted lipolysis means that the thermal damage in the surrounding tissue is likely to be minimal. The findings with this scientific approach to validate clinical results of laser-assisted lipolysis that $1444 \mathrm{~nm}$ wavelength shows the minimal localization of heat over the depth from Monte Carlo simulation for both fat and dermis may be beneficial for other clinical availabilities such as skin rejuvenation, acne treatment, perioral wrinkles and so on.

\section{CONCLUSION}

In this study, the wavelength dependence of laserassisted lipolysis was simulated using the Monte Carlo statistical method with three different wavelengths, $\lambda$ $=1064 \mathrm{~nm}, 1320 \mathrm{~nm}$, and $1444 \mathrm{~nm}$. The simulation was performed with homogeneous fat and dermal tissue, and the heat generation for both types of tissue over the penetration depth was investigated. The results show that the greatest amount of heat generation is observed in both types of tissue at $\lambda=1444 \mathrm{~nm}$, followed by, in order, $\lambda=1320 \mathrm{~nm}$ and $1064 \mathrm{~nm}$. Consequently, $\lambda$ $=1444 \mathrm{~nm}$ shows the highest ablation efficiency, with minimal localization of heat over the depth, when compared with the other two wavelengths, $\lambda=1320 \mathrm{~nm}$ and $1064 \mathrm{~nm}$, for laser-assisted lipolysis.

\section{REFERENCES}

1. D. B. Apfelberg, S. Rosenthal, J. P. Hunstad, B. Achauer, and P. B. Fodor, "Progress report on multicenter study of laser-assisted liposuction," Aesthetic Plast. Surg. 18, 259-264 (1994).

2. D. B. Apfelberg, "Results of multicenter study of laserassisted liposuction,” Clin. Plast. Surg. 23, 713-719 (1996).

3. R. R. Anderson, W. Farinelli, H. Laubach, D. Manstein,

\section{Tiny MC(fat)}

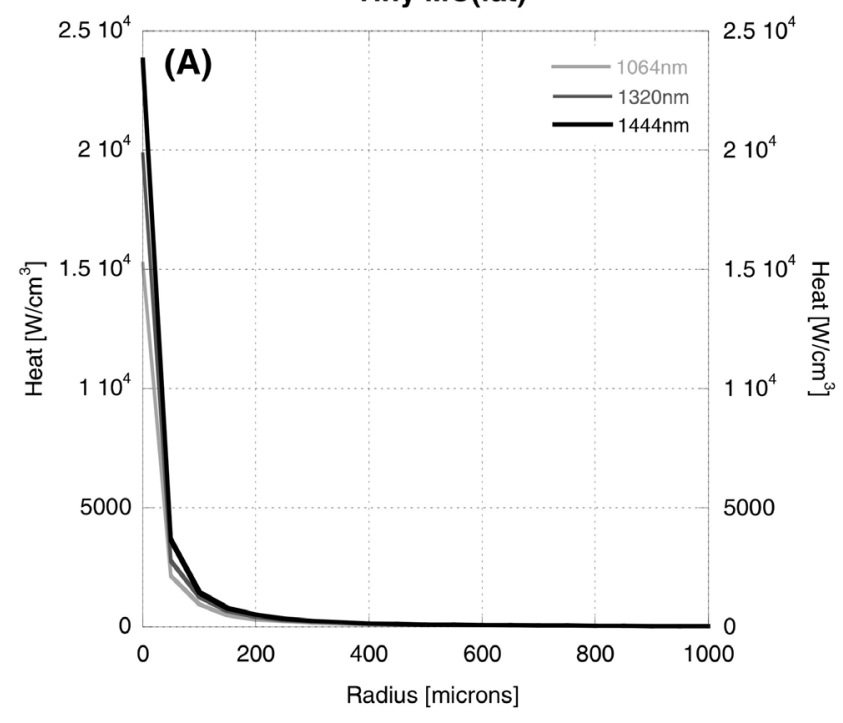

\section{Small MC (fat)}

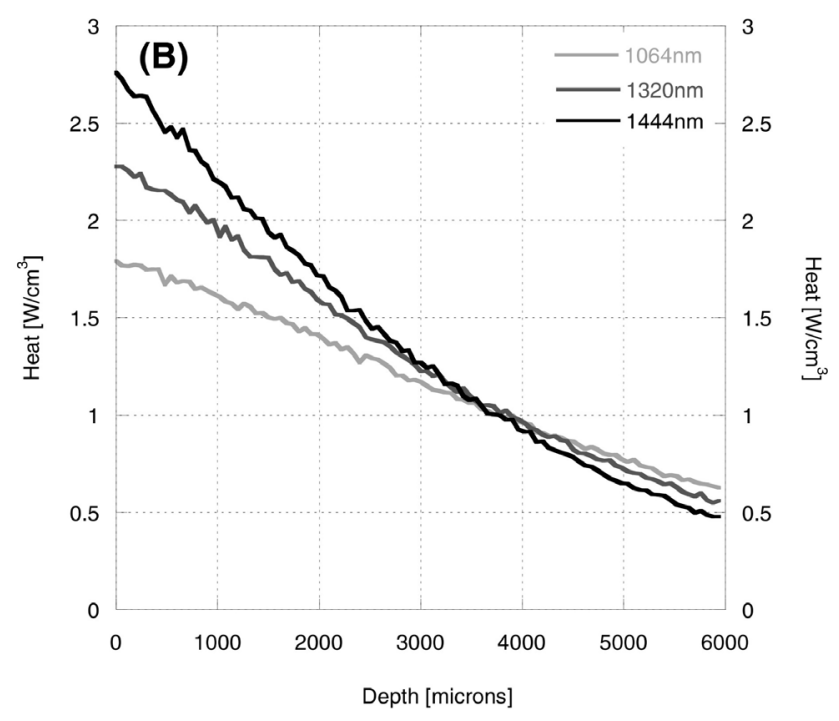

FIG. 2. Relative heat generation vs. penetration depth in fat tissue using Monte Carlo (MC) simulations; (upper) Tiny MC, (lower) Small MC. 
A. N. Yaroslavsky, J. Gubeli, K. Jordan, G. R. Neil, M. Shinn, W. Chandler, G. P. Williams, S. V. Benson, D. R. Douglas, and H. F. Dylla, "Selective photothermolysis of lipid-rich tissues: a free electron laser study," Lasers Surg. Med. 38, 913-919 (2006).

4. J. G. Khoury, R. Saluja, D. Keel, S. Detwiler, and M. P. Goldman, "Histologic evaluation of interstitial lipolysis comparing a 1064, 1320 and $2100 \mathrm{~nm}$ laser in an ex vivo model," Lasers Surg. Med. 40, 402-406 (2008).

5. J. I. Youn, "Evaluation of morphological changes in degenerative cartilage using 3-D optical coherence tomography,” J. Opt. Soc. Korea 12, 98-102 (2008).

6. A. J. Welch and M. J. C. van Gemert, Optical-thermal Response of Laser-irradiated Tissue (Plenum Press, New York, NY, USA, 1995).

7. L. Wang, S. L. Jacques, and L. Zheng, "Monte Carlo modeling of light transport in multi-layered tissues,"
Computer Methods and Prog. Biomed. 47, 131-146 (1995). 8. S. A. Prahl, M. Keijzer, S. L. Jacques, and A. J. Welch, "A Monte Carlo model of light propagation in tissue," Proc. SPIE Institute Series IS 5, 102-111 (1989).

9. A. N. Bashkatov, E. A. Genina, V. I. Kochubey, and V. V. Tuchin, "Optical properties of the subcutaneous adipose tissue in the spectral range 400-2500nm," Geometrical and Applied Optics 99, 868-874 (2005).

10. S. R. Mordon, B. Wassmer, J. P. Reynaud, and J. Zemmouri, "Mathematical modeling of laser lipolysis," Biomed. Eng. Online 7, 10 (2008).

11. A. J. Welch and C. M. Gardner, "Monte Carlo model for determination of the role of heat generation in laserirradiated tissue," J. Biomech. Eng. 119, 489-495 (1997).

12. Y. H. Ju, J. H. Park, J. H. Lee, J. Y. Lee, K. B. Nahm, and J. H. Kim, "Study on the simulation model for the optimization of optical structures of edge-lit backlight for LCD applications," J. Opt. Soc. Korea 12, 25-30 (2008). 